\title{
Density of Ultra-Pure Air at 298.15 K for Mass Transfer Buoyancy Corrections
}

\author{
Meyer Waxman and Harry Davis \\ Center for Themodynamics and Molecular Science, National Bureau of Standards \\ Washington, DC 20234
}

\begin{abstract}
June 27,1978
Within the context of the general problem to attain improved accuracy for the transfer of apparent mass values, the PVT properties of ultrapure air at $298.15 \mathrm{~K}$ for low pressures have been determined. At 0.1 MPa, the accuracy of the molar density is estimated to be 0.001 percent. Our application of the isothermal Burnett method to obtain the "PVT" measurements, the analysis of these measurements, and the effects of systematic Burnett errors on the PVT results are discussed.
\end{abstract}

Key words: Burnett method; low pressures; PVT; ultra-pure air; $298.15 \mathrm{~K}$.

The accurate transfer of an apparent mass value from one object to another is made with a balance comparison of the objects in a gaseous environment, usually the atmosphere, and consequently is affected by the accuracy with which the buoyant force acting on each object is known. The buoyancy itself is proportional to the density of the gas and the volume of the gas displaced by the object; thus the accuracy of the net buoyancy correction to the compared masses depends on the errors associated with the density of the gas and the volume difference between the objects. In the traditional mass transfer, a major error in the buoyance correction is attributed to the uncertainty of the knowledge of the pressurevolume-temperature (denoted by PVT) properties of the gas $[1,2] .{ }^{1}$ The effect of this error, which we discuss further, limits the current accuracy of mass transfer. For example, in the mass comparison of two kilogram-objects fabricated from materials with the contrasting densities, aluminum $(2700 \mathrm{~kg} /$ $\mathrm{m}^{3}$ ) and stainless-steel $\left(7800 \mathrm{~kg} / \mathrm{m}^{3}\right)$, the buoyancy correction for atmospheric air of normal composition would be equivalent to $0.29 \mathrm{~g}$. If the resolution of the balance used for the transfer were comparable with that of kilogram balances in national laboratories, $0.005 \mathrm{ppm}$ or $5 \mu \mathrm{g}$, the buoyancy correction for the ultimate accuracy would have to be known to 0.002 percent. This accuracy is not achieved in practice. Usually the errors associated with the measurement of the buoyancy variables-moisture content, pressure, temperature, and volume of the displaced air - limit the accuracy of apparent mass comparisons for kilogram objects of contrasting densities to about two orders of magnitude less than the maximum balance resolution. Even for a mass transfer precision of $0.1 \mathrm{ppm}$ in the atmosphere, the buoyancy accuracy of 0.03 percent needed in this example would

\footnotetext{
${ }^{1}$ Figures in brackets indicate literature references at the end of this paper.
}

require that the moisture contribution in the density of the air, typically 1 percent, be itself known to 3 percent.

The present transfer limitation imposed by buoyancy uncertainties suggests that mass transfers be made with a balance operated in a chamber which is either sufficiently evacuated to reduce the buoyancy correction to a negligible magnitude or which is filled with a gas whose density is accurately known as a function of the operating pressure and temperature. However the transition to such environments from an atmospheric one without a significant loss of accuracy still requires further substantiation of the PVT properties of atmospheric air. For this reason, and in particular to evaluate the reliability for literature values [3], we have redetermined the molar density of dry air at $298.15 \mathrm{~K}$ for low pressures. Our work also serves to indicate the accuracy with which the molar density of a suitable environmental gas can be determined. In this paper we present the results and our evaluation of them.

For this redetermination we have used a Burnett PVT apparatus, schematically shown in figure 1 , which we have developed and used for the accurate determination of the PVT properties (defined as the compressibility factor) of gases over a wide range of conditions [4]. The Burnett method itself, is an experimentally simplified method in which neither the quantity of gas nor its volume is measured; instead accurate isothermal pressure measurements are made before and after stepwise expansions between two volumes. The measured pressures are converted into a known compressibility factor, defined further as a pressure or density series, through data reduction involving the least-squares evaluation of parameters in Burnett nonlinear equations. Details of the data reduction have been published elsewhere [5]. 


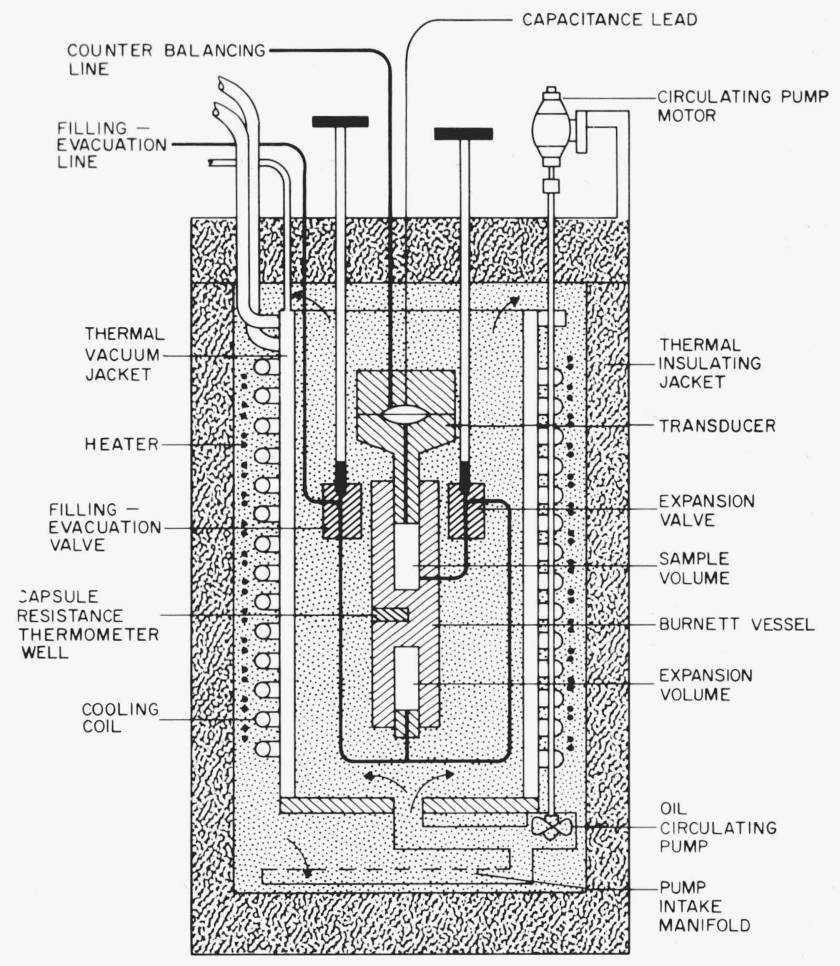

Figure 1. Burnett PVT apparatus
Because the equations needed in the data reduction are nonlinear, the interpretation of results is subtle. The reliability of results can be estimated, however, from the smallness of the standard deviation, the invariance of the volume ratio parameter as evaluated from data obtained for gases with different nonideality behaviors, and a study of the effects of systematic errors. For these reasons, we report data for both helium and dry air, given in table 1 , and also discuss the effects of Burnett systematic errors.

In our experiments the pressure measurements are made with a combination of a controlled-clearance piston gauge, with gas as its pressure fluid, and a mercury barometerboth primary standards. The gas being studied is confined to the Burnett volumes and separated from the pressure measuring instrumentation by a closely-coupled, sensitive, and highly-reproducible differential pressure transducer. This allows all of the sample gas to be readily thermostated to within $0.002 \mathrm{~K}$ of the required isotherm temperature. The measured pressure corresponds to that of the counterbalancing gas used to null the transducer. The accuracy and precision of the pressure measurements are optimum for pressures from $0.3 \mathrm{MPa}$ to $3.7 \mathrm{MPa}$. For this range, the accuracy and precision are 0.003 percent and 0.001 percent or better. Under various experimental conditions, we have found that a pressure measurement usually repeats to better than 0.001 percent; if it does not we consider the disagree-

TABLE 1.

Results of nonlinear statistical analysis for dry-air Burnett PVT data. ${ }^{a-c}$

\begin{tabular}{|c|c|c|c|c|}
\hline & Helium & & Air & \\
\hline Compressibility factor & $\left(1+b_{2} P+b_{3} P^{2}\right)$ & & $\left(1+B_{2} \rho+B_{3} \rho^{2}\right)$ & \\
\hline Volume ratio status & single parameter & constant & single parameter & $\begin{array}{l}\text { a parameter/each data } \\
\text { set }\end{array}$ \\
\hline value & 1.78070 & 1.78070 & 1.78068 & $\begin{array}{l}1.78068(\text { set } 1) \\
1.78068(\text { set } 2)\end{array}$ \\
\hline uncertainty & $0.00000_{6}$ & & 0.00003 & $\begin{array}{l}0.00003(\text { set } 1) \\
0.00003(\text { set } 2)\end{array}$ \\
\hline $\begin{array}{l}\text { Second virial coefficient } \\
\text { value } \\
\text { uncertainty }\end{array}$ & $\begin{array}{l}\mathrm{MPa}^{-1} \\
4.795 \times 10^{-3} \\
0.005 \times 10^{-3}\end{array}$ & $\begin{array}{l}\mathrm{cm}^{3} / \mathrm{mol} \\
-7.45 \\
0.02\end{array}$ & $\begin{array}{l}\mathrm{cm}^{3} / \mathrm{mol} \\
-7.38 \\
0.11\end{array}$ & $\begin{array}{l}\mathrm{cm}^{3} / \mathrm{mol} \\
-7.37 \\
0.12\end{array}$ \\
\hline $\begin{array}{l}\text { Third virial coefficient } \\
\text { value } \\
\text { uncertainty }\end{array}$ & & $\begin{array}{l}\mathrm{cm}^{6} / \mathrm{mol}^{2} \\
1293 \\
16\end{array}$ & $\begin{array}{l}\mathrm{cm}^{6} / \mathrm{mol}^{2} \\
1267 \\
45\end{array}$ & $\begin{array}{l}\mathrm{cm}^{6} / \mathrm{mol}^{2} \\
1262 \\
50\end{array}$ \\
\hline Standard deviation $(\Delta P / P)$ & $0.5 \times 10^{-5}$ & $0.7_{7} \times 10^{-5}$ & $0.8_{2} \times 10^{-5}$ & $0.8_{7} \times 10^{-5}$ \\
\hline $\begin{array}{l}\mathrm{Z}(3.5232 \mathrm{MP}) \\
\text { uncertainty }\end{array}$ & & $\begin{array}{l}0.99198 \\
0.00000_{8}\end{array}$ & $\begin{array}{l}0.99203 \\
0.00007\end{array}$ & $\begin{array}{l}0.99203 \\
0.00008\end{array}$ \\
\hline$Z(0.30029 \mathrm{MPa})$ & & 0.99912 & 0.99912 & 0.99912 \\
\hline uncertainty & & $0.00000_{3}$ & 0.00001 & 0.00001 \\
\hline$Z(0.1 \mathrm{MPa})$ & & 0.99970 & 0.99970 & 0.99970 \\
\hline
\end{tabular}

${ }^{a}$ Gas constant $=8.3143_{5}\left(\mathrm{MPa} \mathrm{cm}{ }^{3} \mathrm{~mol}^{-1} \mathrm{~K}^{-1}\right)$.

${ }^{b}$ Temperature $=298.150 \mathrm{~K}$.

${ }^{c}$ The pressure is denoted by $P$, the pressure virial coefficients by $b_{i}$, the density by $\rho$, the density virial coefficients by $B_{j}$, and the compressibility factor by $Z$. 
ment to be indicative of an apparatus malfunction or chemical instability of the sample gas and do not accept the measurement as a valid data point. Further, the experiment is not continued until the fault is corrected. The sample gas temperature as defined by the International Practical Temperature Scale of 1968 is measured with a capsule platinum thermometer located in the wall of the Burnett vessel.

Our sample of ultra-pure air was especially purified by Scott-Marrin Inc. [6] and supplied in an anodized aluminum cylinder designed to preserve the purity of the gas. The carbon dioxide content of the air was, initially, removed during its purification; afterwards, 0.032 mole percent of carbon dioxide was added by the supplier to the purified air. The sample air was analyzed with a mass spectrometer by the Air and Water Pollution Analysis Section of the National Bureau of Standards. Its composition approximates that for dry atmospheric air to within 0.03 mole percent; the analysis results for the principal constituents are:

$$
\begin{array}{lllll}
\mathrm{N}_{2} & \mathrm{O}_{2} & \mathrm{Ar} & \mathrm{CO}_{2} & \mathrm{Air}
\end{array}
$$

Composition, mole 78.1220 .920 .930 .032

$$
\text { percent }
$$

Molecular weight, $\mathrm{kg} /$

0.028938

mol

Minor constituents such as $\mathrm{Kr}, \mathrm{Ne}$, and $\mathrm{Xe}$ are assumed to be present in normal quantities. Scott-Marrin's estimate of contaminants (ppm per volume) in the sample prior to the addition of the carbon dioxide are water $<5$, total $\mathrm{H}_{\mathrm{x}} \mathrm{C}_{\mathrm{xx}}<$ $0.01, \mathrm{CO}<0.01, \mathrm{NO}_{\mathrm{x}}<0.01$ and $\mathrm{CO}_{2}<0.001$. To preserve the sample quality during the experiment, the apparatus was evacuated and flushed with the source gas prior to the initial filling of the sample volume; further, independent measurements were made with different samples from the same cylinder. Helium gas of 99.999 percent purity was used to obtain complementary Burnett PVT data to substantiate the value of the volume ratio parameter obtained from the air PVT data.

Two sets of Burnett PVT data were obtained at 298.15K for both the ultra-pure air and the helium. Each set consisted of data from a series of four consecutive expansions from the highest pressure to the lowest pressure compatible with the optimum precision and accuracy of the measurements. The initial pressure of the second set for each gas was selected so as to intersperse the data of the second set with those of the first set. For the air data, the compressibility factor was represented by a second degree density series, which contributed two parameters - the second and third density virial coefficients - to the overall parameter evaluation. For helium, a second degree pressure series was used; the value of the third virial coefficient was obtained from literature values rather than from our experimental data. The contribution of this term to the compressibility factor is so small for our pressures that the other parameters are more accurately evaluated with one fewer parameter.

Results are given in table 1 for the nonlinear least-squares evaluation of the Burnett equations formed from all of the helium data and, similarly, from all of the air data. In the helium data analysis, the parameters are common to all of the data; whereas, for the air data analysis, an initial density parameter is designated for each air data set and the role of the volume ratio parameter is varied for three different cases. In the first case, the volume ratio parameter is considered to have a constant value as determined from the helium data analysis; in the second, it is considered to be single parameter common to all of the air data; and in the third case, a volume ratio parameter is designated for each air data set. In any of our Burnett data analyses, all of the parameters pertaining to a particular analysis are simultaneously evaluated together with the "true" values of the measured pressures for the analysis.

To facilitate mass transfer buoyancy corrections, we have generated density and compressibility factor values for the pressure range of probable interest from our air density virial coefficients. These results are presented in table 2 along with comparative values obtained from "Tables of Thermal Properties of Gases" by J. Hilsenrath et al. [3]. The pressure interval used in table 2 is small enough to allow for linear interpolation. The literature values differ from ours by as little as 0.001 percent at $0.1 \mathrm{MPa}$. At higher pressures, not listed, the agreement is not as favorable, there being as much as 0.04 percent difference at $3.4 \mathrm{MPa}$. For temperature differences less than $5 \mathrm{~K}$ from the reference temperature of $298.150 \mathrm{~K}$, a change in density with respect to a change in temperature for the same low pressures may be approximated with sufficient reliability from PVT correlation data for dry air given in reference [3].

TABLE 2.

Pressure, density, and compressibility factor values for air at $298.150 \mathrm{~K}$.

\begin{tabular}{ccccc}
\hline $\begin{array}{c}\text { Pressure } \\
(\mathrm{MPa})\end{array}$ & \multicolumn{2}{c}{$10^{4} \times$ Density } & \multicolumn{2}{c}{ Compressibility factor } \\
\hline & this paper & reference 3 & this paper & reference 3 \\
\hline 0.01 & 0.040341 & 0.040341 & 0.99997 & 0.99997 \\
.02 & .080686 & .080684 & .99994 & .99994 \\
.03 & .121030 & .121030 & .09991 & .99991 \\
.04 & .161379 & .161379 & .99988 & .99987 \\
.05 & .201729 & .201730 & .99985 & .99984 \\
.06 & .242083 & .242083 & .99982 & .99981 \\
.07 & .282438 & .282439 & .99979 & .99978 \\
.08 & .322796 & .322798 & .99976 & .99975 \\
.09 & .363156 & .363159 & .99973 & .99972 \\
.10 & .403519 & .403523 & .99970 & .99969 \\
.11 & .443884 & .443889 & .99967 & .99965 \\
.12 & .484251 & .484258 & .99964 & .99962 \\
.13 & .524621 & .524629 & .99961 & .99959 \\
.14 & .564993 & .565002 & .99958 & .99956 \\
.15 & .605368 & .605378 & .99955 & .99953 \\
\hline
\end{tabular}


The estimation of accuracy for Burnett results is necessarily somewhat subjective since systematic errors do not propagate directly in the Burnett equations. The nonlinear statistical uncertainties reflect the quality of the experimental data relative to the particular model - the Burnett equation used to describe the experiment analytically. The values obtained for the virial coefficients depend slightly upon whether the volume ratio is determined simultaneously with the other parameters or is assumed to be known from the helium experiments. This variation arises from three sources: the higher order nonideality behavior of the sample gas, the truncation of the series, and the inevitable high statistical correlation between parameters. For our reported data, however, the effect on the series value, i.e. the compressibility factor, is quite small for our pressure region of interest, namely less than 0.001 percent. The effects of systematic errors are preferably studied by the analysis of reliable Burnett data with simulated systematic errors superimposed on the data. We have discussed such an analysis in an earlier paper [5]; some of the pertinent results are given in table 3. The effects of similar errors in the analysis of our Burnett air data should be no greater than for those given in table 3. The negligible effects of the systematic and random errors on the compressibility factor at the low pressures are a consequence of the approximation to its fixed limit, i.e., the theoretical limit of unity as the density approaches zero.

TABLE 3.

Effects of systematic errors superimposed on $\mathrm{C}_{2} \mathrm{H}_{4}$ Burnett PVT data at $273.15 \mathrm{~K}$

\begin{tabular}{lcc}
\hline \hline \multicolumn{1}{c}{ Error source } & Percentage error & $\begin{array}{c}\text { Percentage change in Z } \\
(0.1 \mathrm{MPa})\end{array}$ \\
\hline Volume ratio & 0.0011 & $<0.0005$ \\
Pressure & 0.003 & $<0.0005$ \\
RT & 0.005 & $<0.0005$ \\
\hline
\end{tabular}

We conclude that the molar density of dry air presented here would allow for an apparent mass balance comparison between aluminum and stainless-steel kilogram objects to an accuracy better than $0.005 \mathrm{ppm}$ in a pure dry air environment. Slight variations in the composition of the air used from that of our sample air would have only a negligible effect on the PVT results. The practical realization of this accuracy will, of course, depend upon any additional errors incurred in the measurement accuracies of the pressure, temperature, and composition of the buoyancy gas environment. It will also be affected by the accuracy of the values used for the gas constant and for the molecular weights of the constituents of the pure air. Ideally, one would want the limiting accuracy to be determined by the uncertainties associated with the values for the molecular weights. For air, these have been estimated to correspond to a total uncertainty of 0.001 percent of the molecular weight of the air environment.

\section{References}

[1] Pontius, P. E. Science 190, 379 (1975).

[2] Diehl, H., Koch, W., Davis, R. S. and Bower, V. E. A Device for Invacuo Mass Comparisons of Artifacts for Direct Air Density Determination, J. Res. Nat. Bur. Stand. (U.S.), 83, No. 5, (Sept.Oct. 1978).

[3] Tables of Thermal Properties of Gases, Natl. Bur. Stand. (U.S.), Circular 564 (1955).

[4] Waxman, M. and Hastings, J. R. J. Res. Nat. Bur. Stand. (U.S.), 75C (Eng. and Instr.) No. 2, 79-83, (April-June 1971).

[5] Waxman, M., Davis, H. A., and Hastings, J. R. Symp. Thermophy. Properties, 6th, 245 (1973).

[6] In order to describe materials and experimental procedures adequately, it is occasionally necessary to identify commercial products by manufacturer's name. In no instance does such identification imply endorsement by the National Bureau of Standards, nor does it imply that the particular product is necessarily the best available for that purpose. 